\title{
Reliable Mobility Evaluation of Organic Field-Effect Transistors with Different Contact Metals
}

\author{
Fanming Huang, Ao Liu, Huihui Zhu, Yong Xu, Member, IEEE, Francis Balestra, Senior Member, \\ IEEE, Gerard Ghibaudo, Fellow, IEEE, Yong-Young Noh, Junhao Chu, and Wenwu Li
}

\begin{abstract}
The reliability of mobility evaluation for organic field-effect transistors (OFETs) has recently been a serious issue since numerous overestimated and underestimated mobilities have been reported. A reliable approach to accurately evaluate the OFET mobility is highly desirable. Under this background, two commonly used methods and the Y function method (YFM) are used to extract the mobility of indacenodithiophene -co-benzothia-diazole (IDT-BT) OFETs with four different contact metals. It is observed that the mobilities extracted by other methods vary greatly with the contact metal and the channel length, while the mobility extracted by YFM is very stable and approaches the inherent mobility of IDT-BT. Our results show that YFM can be a precise alternative to be widely used for OFET mobility evaluation.
\end{abstract}

Index Terms-Mobility, characterization, OFETs, contact resistance.

\section{INTRODUCTION}

M obility, determining how quick the charges move through the channel and thus the output driving current, is a key performance parameter of organic field-effect transistors (OFETs). Typically, OFET mobility is extracted by the conventional method based on the saturation transfer characteristics [1], [2]. However, that model was initially developed for silicon metal-oxide-semiconductor field-effect transistors (MOSFETs), which is for gradual channel approximation with ohmic contacts and band transport [3].

Manuscript received December **, 2018. This work was financially supported by National Key Research and Development Program of China (Grant No. 2016YFB0501604), Natural Science Foundation of China (Grant Nos. 61774061 and 61504043), the NSAF foundation (Grant No. U1830130), East China Normal University Scholarship Program for Graduate Students, the Center for Advanced Soft-Electronics (2013M3A6A5073183) funded by the Ministry of Science \& ICT through the NRF grant funded by the Korea government, and the 2018 Government-funded Research Program of the Korea Institute of Machinery and Materials (NK210D).

F. Huang, J.H. Chu, and W.W. Li are with Key Laboratory of Polar Materials and Devices (Ministry of Education), Department of Electronic Engineering, East China Normal University, Shanghai 200241, China. (e-mail: wwli@ee.ecnu.edu.cn ).

Y. Xu is with School of Electronic and Optical Engineering, Nanjing University of Posts and Telecommunications, Nanjing, Jiangsu, 210023, China. (e-mail:xuyong@njupt.edu.cn).

A. Liu, H.H. Zhu, Y.Y. Noh are with Department of Energy and Materials Engineering, Dongguk University, 30 Pildong-ro, 1-gil, Jung-gu, Seoul 04620, Republic of Korea. (e-mail: yynoh@ dongguk.edu ).

F. Balestra and G. Ghibaudo are with the Institut de Microélectronique Electromagnétisme et Photonique and Laboratoire d'Hyperfréquences et de Caractérisation, Grenoble Institute of Technology, Micro and Nanotechnology Innovation Centre, F-38016 Grenoble, France.
OFETs, on the other hand, are generally with disordered organic semiconductor and mismatched energy levels for charge injection. This gives rise to variable-range hopping charge transport and very high contact resistance [4], [5], leading to severe deviations from the ideal model. As a consequence, the mobilities of those OFETs can be significantly overestimated or underestimated [1], [6], [7]. It is thus highly desirable to find a reliable approach to evaluate the inherent carrier mobility.

The $\mathrm{Y}$ function method (YFM) was first established for MOSFET parameter extraction [8], in particular, the low-field mobility and contact resistance. Differing from the conventional method, YFM applies in the linear regime (i.e. in the low-field regime) and, most importantly, it takes into account the influence of the contact resistance. So, YFM is free from extrinsic effects and has demonstrated much superior reliability [9]. Nevertheless, a work reporting how to reliably extract mobility without the contact metal effect is still lacking.

In this letter, polymer OFETs using state-of-the-art polymer semiconductor of indacenodithiophene-co-benzothiadiazole (IDT-BT) are fabricated with various contact metals. Three different extraction methods are adopted to evaluate their mobilities. Different trends are observed when plotting the corresponding mobilities against contact metal. The dependence of mobility on the contact resistance and the channel length are discussed. The mobility extracted by YFM proves to be more accurate and inherent as compared to other mobilities extracted by the commonly used methods. Thus, YFM turns out to be a reliable approach to evaluate OFET mobility.

\section{DEVICE FABRICATION AND CHARACTERIZATIONS}

In this work, top-gate staggered IDT-BT transistors were fabricated (Fig. 1(a)). The drain and source electrodes were formed by thermal evaporation with four different contact metals ( $\mathrm{Au}, \mathrm{Ag}, \mathrm{Cu}$, and $\mathrm{Ni}$ ) on the pre-cleaned glass substrate via designed shadow masks. The channel width $(W)$ was fixed to $1000 \mu \mathrm{m}$ with the length $(L)$ varying from $60 \mu \mathrm{m}$ to $350 \mu \mathrm{m}$. IDT-BT was dissolved in dichlorobenzene $(10 \mathrm{mg} / \mathrm{ml})$ and was spin-coated at $2000 \mathrm{rpm}$ for $60 \mathrm{~s}$, and then the samples were annealed at $100{ }^{\circ} \mathrm{C}$ for 1 hour in a nitrogen-filled glovebox. Subsequently, the IDT-BT layer was patterned by lithography to suppress the leakage. Afterward, polystyrene, as the first dielectric layer, dissolved in n-butyl acetate $(80 \mathrm{mg} / \mathrm{ml})$ was 
spin-coated at $2000 \mathrm{rpm}$ for $60 \mathrm{~s}$ and then annealed at $80{ }^{\circ} \mathrm{C}$ for 2 hours. The second dielectric layer of Cytop ${ }^{\mathrm{TM}}$ was spin-coated at $2000 \mathrm{rpm}$ for $60 \mathrm{~s}$ and then annealed at $80{ }^{\circ} \mathrm{C}$ for 1 hour. Finally, the top-gate Au electrode was made by thermal evaporation by using shadow masks. The electrical characterizations were performed in nitrogen atmosphere using a semiconductor parameter analyzer (Keithley 4200-SCS).
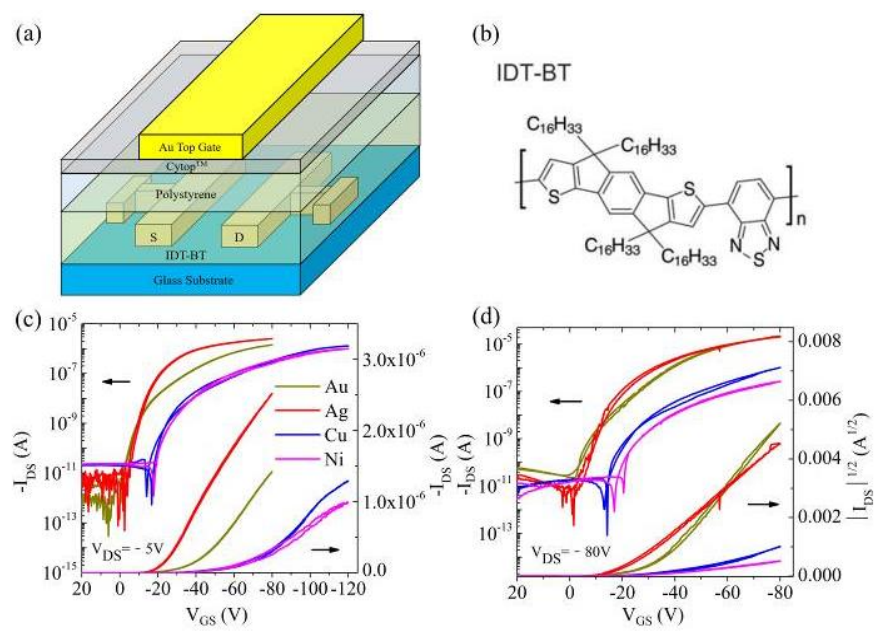

Fig .1. (a) Schematic illustration of the top-gate staggered IDT-BT OFETs. The contact metal is $\mathrm{Au}, \mathrm{Ag}, \mathrm{Cu}$, and $\mathrm{Ni}$, respectively. (b) Chemical structure of IDT-BT. (c) Linear and (d) saturation transfer characteristics of the IDT-BT OFETs with four different contact metals.

\section{RESULTS AND DISCUSSIONS}

The typical transfer characteristics are shown in Fig. 1(c) and 1(d) for the four contact metals. One can observe a large discrepancy even though the semiconductor and dielectric are unchanged. An obvious kink, which has been considered to be a sign of the nonideality, exists in the OFETs with $\mathrm{Cu}$ and $\mathrm{Ni}$ contact metals, especially in the linear regime [10]. It means that these transistors suffer from extrinsic effects when contact metal varies. The reliability of the mobilities extracted simply by the transfer characteristics can be disturbed.

To further investigate the mobility, three extraction methods are discussed for comparison. They can be expressed by the following three equations [8], [11]: for the saturation mobility $\mu_{S A T}=2 L / W C_{O X} \cdot\left(\partial\left(I_{D S S A}\right)^{1 / 2} / \partial V_{G S}\right)^{2}$, and for the effective mobility $\mu_{L I N}=L / V_{D S} W C_{O X} \cdot \partial I_{D S L I} / \partial V_{G S}$, and for the low-field mobility by the $\mathrm{Y}$ function method $\mu_{Y}=L / V_{D S} W C_{O X}$ $\cdot \partial\left(I_{D S L I}\left(\partial I_{D S L I} / \partial V_{G S}\right)^{-1 / 2}\right) / \partial V_{G S}$, where $I_{D S S A}, I_{D S L I}, V_{D S}, V_{G S}, L, W$, and $C_{O X}$ are the drain current in the saturation regime, the drain current in the linear regime, the drain voltage, the gate voltage, the channel length, the channel width, and the areal gate dielectric capacitance. The three mobilities extracted by these methods are plotted in Fig. 2, where the error bars represent the standard deviation calculated from more than 10 samples. Obviously, $\mu_{Y}$ is stable and independent of the contact metal.

By contrast, the mobilities extracted by the other two methods $\left(\mu_{S A T}\right.$ and $\left.\mu_{\text {LIN }}\right)$ vary greatly with the contact metal. Especially, $\mu_{S A T}$ decreases nearly two orders of magnitude from $2.116 \mathrm{~cm}^{2} / \mathrm{V} \cdot \mathrm{s}$ to $\left(0.03\right.$ ?) $\mathrm{cm}^{2} / \mathrm{V} \cdot \mathrm{s}$ when the contact metal changes from $\mathrm{Au}$ to $\mathrm{Ag}, \mathrm{Cu}$, and $\mathrm{Ni}$. Compared to the previous mobility values reported for IDT-BT OFETs [12], [13], which are around $1 \mathrm{~cm}^{2} / \mathrm{V} \cdot \mathrm{s}$, so the mobility here is overestimated for $\mathrm{Au}$ contacted OFETs while is severely underestimated for $\mathrm{Cu}$ or Ni contacted OFETs.

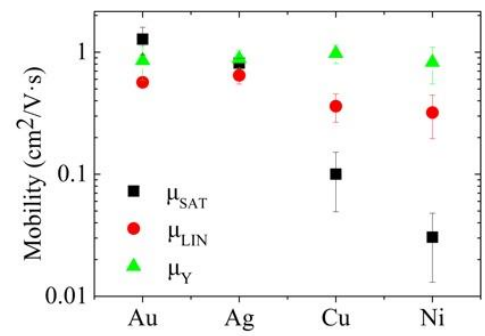

Fig .2. The mobilities extracted by different methods with the $\mathrm{Au}, \mathrm{Ag}, \mathrm{Cu}$, and $\mathrm{Ni}$ contact metal, respectively.

Differing from the other two mobilities, $\mu_{S A T}$ is extracted only from the saturation regime (i.e. at high drain voltages). High drain voltage can produce high output driving current thus alleviating the mobility underestimation induced by contact resistance, and sometimes very high mobility can be obtained [14]. However, such superficial values can be affected by the channel length modulation [15], hopping barrier lowering [16] etc., which exist in the OFETs under very high lateral field. The former effect can lead to misestimated channel length owing to the source-oriented shift of the pinch-off point. The latter effect causes a reduced hopping barrier and thus the extracted mobility deviates from the inherent value [16], [17]. These may account in part for the higher $\mu_{S A T}$ when the OFETs use Au as contact metal.
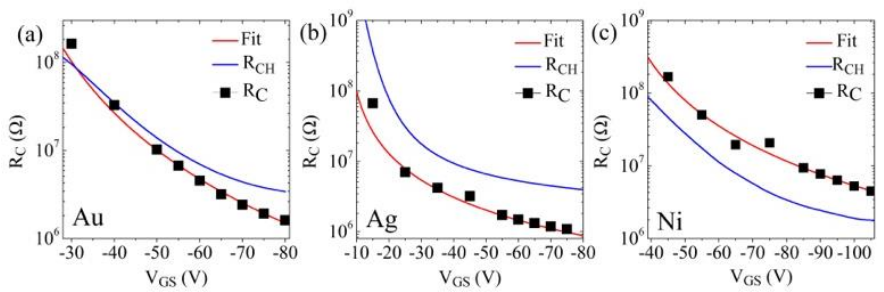

Fig .3. The contact resistance $\left(\mathrm{R}_{\mathrm{C}}\right)$ and channel resistance $\left(\mathrm{R}_{\mathrm{CH}}\right)$ estimated at different gate voltage.

On the other side, the underestimated $\mu_{S A T}$ for $\mathrm{Cu}$ and $\mathrm{Ni}$ contacted OFETs, and the variation of $\mu_{\text {LIN }}$ extracted from the linear regime, are also observed. Actually, these results stem from the contact resistance, as reported extensively before [2], [18], [19]. Due to the lack of a matured doping technique for organic semiconductor, ohmic contacts are difficult to form in OFETs. As a result, the contact resistance $\left(R_{C}\right)$ that often comes from very high Schottky barrier has been a main reason for the non-ideal characteristics of many OFETs. We thus extract $R_{C}$ by the transfer-length method (TLM) and compare it with the channel resistance $\left(R_{C H}\right)$, as seen in Fig. 3.

For better illustration, an empirical formula is employed to fit the extracted $R_{C}$ and the fitting is illustrated by red line [11]. One can see that, the $R_{C}$ decreases more rapidly than $R_{C H}$ for $\mathrm{Au}$ contacted transistors, while $R_{C}$ is always dominant in $\mathrm{Ni}$ contacted OFETs. The resistance evolvement of $\mathrm{Cu}$ contacted transistors is similar to Ni (not shown here). Combined with the mobility summarized in Fig. 2, our result is consistent with the 
model proposed by Liu et al. about gate-voltage dependent resistance [2], where the Au contacted situation corresponds with the overestimated mobility and the Ni contacted situation is vice versa. It also proves that $\mu_{S A T}$ and $\mu_{L I N}$ extracted from $R_{C}$-dominant OFETs $\left(R_{C}>R_{C H}\right.$ or $\left.R_{C} \approx R_{C H}\right)$ are seriously disturbed, and therefore the correspondingly extracted mobility are not reliable. Only the mobility extracted from the $R_{C H}$-dominant OFETs $\left(R_{C} \ll R_{C H}\right)$ like Ag contacted OFETs here are relatively reliable. Interestingly, the mobility of $\mathrm{Ag}$ contacted OFETs is almost independent of the extraction method (Fig. 2).
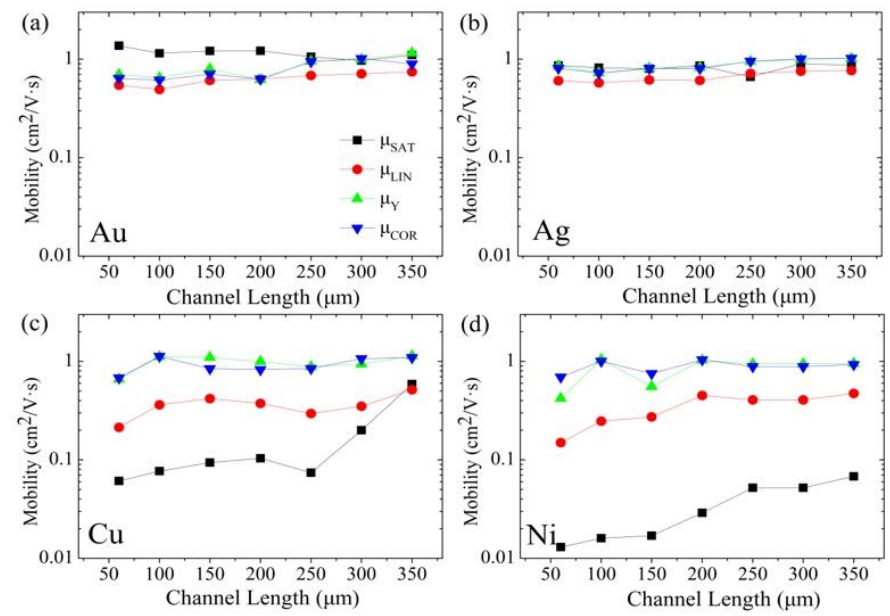

Fig .4. The channel length dependence of the different method extracted mobility with respect to $\mathrm{Au}(\mathrm{a}), \mathrm{Ag}(\mathrm{b}), \mathrm{Cu}$ (c) and $\mathrm{Ni}$ (d) contact metal.

Nmerous extrinsic effects have been examined to shed light on the unreliablity of mobility evaluation, yet YFM is insusceptible. Is the stable low-field mobility reliable? In general, if all the extrinsic effects are removed, the mobilities extracted by different methods should be close and approach the inherent mobility of the applied semiconductor. According to Fig. 2, only the mobilities extracted by $R_{C H}$-dominant transistor, i.e., Ag contacted OFETs satisfy this inference. It means that the mobility extracted from Ag contacted OFETs is free from extrinsic effects. From another point of view, the $\mu_{Y}$ is very stable with the contact metal. Note that, this mobility is almost identical to the value of Ag-contacted OFETs and agrees with the literature for IDT-BT OFETs [12], [16]. These results suggest that the $\mu_{Y}$ extracted by YFM is reliable.

To explore $\mu_{Y}$ reliability, the channel mobility $\left(\mu_{o}\right)$ is investigated to compare with $\mu_{Y}$. Here, $\mu_{o}$ is derived from the OFETs with various channel lengths by using TLM(not shown). Thus, $\mu_{o}$ is free from the influence of contact resistance. As expected, one observes a contact independent $\mu_{o}$ whose value is about $0.9-1 \mathrm{~cm}^{2} / \mathrm{V} \cdot \mathrm{s}$ for the four metal contacted OFETs, very close to $\mu_{Y}$. This result indicates that, the intrinsic mobility can be evaluated by YFM. However, YFM requires only a single OFET.

Another notable characteristic of YFM is shown in Fig. 4. $\mu_{Y}$ always remains the inherent value while $\mu_{S A T}$ and $\mu_{L I N}$ significantly vary with the channel length $(L)$ and then approach the constant $\mu_{Y}$. It implies that the mobilities are gradually independent on their extraction methods, i.e., more intrinsic mobilities can be obtained. This is because the contact effect plays an important role in limiting the apparent mobility of the short-channel devices, in which $R_{C}$ is much higher than $R_{C H}$ since $R_{C}$ is almost constant with $L$ while $R_{C H}$ decreases proportionally with shortening $L$. Mobility underestimation is thus inevitable for the short-channel transistors [22], [23]. To further verify this inference, the previous $\mu_{L I N}$ is corrected for $R_{C}$, which can be done by correcting the measured drain current with the $R_{C}$ and $R_{C H}$, i.e., $I_{D S C O R}=I_{D S L I}\left(1+R_{C} / R_{C H}\right)$ [21], where $I_{D S C O R}$ represents the drain current without contact effects. The corrected mobility $\left(\mu_{C O R}\right)$ is replotted in Fig. 4, where $\mu_{C O R}$ and $\mu_{Y}$ are nearly identical and independent of the contact metal as well as the channel length. Given this result, it can be concluded that the largely varying $\mu_{\text {LIN }}$ with the contact metal is due to the contact resistance. Only the long-channel transistors are reliable for extracting mobility if the contact resistance is high. From a practical point of view, one does not know what is the adequate channel length for this purpose. A better way is to apply YFM that directly offers the inherent $\mu_{Y}$ independent of the contact metal and the channel length, .

\section{CONCLUSIONS}

Three mobility extraction methods are employed to analyze the OFETs with four different contact metals. YFM proves to be the most reliable method to evaluate mobility since it directly provides the inherent mobility independent of the contact metal and the channel length. By contrast, the widely used methods give $\mu_{L I N}$ and $\mu_{S A T}$ that greatly vary with the contact metal and the channel length, leading to large mobility misestimation. Therefore, YFM can be a reliable alternative to extract OFET mobility.

\section{REFERENCES}

[1] A. F. Paterson, S. Singh, K. J. Fallon, T. Hodsden, Y. Han, B. C. Schroeder, H. Bronstein, M. Heeney, I. McCulloch, and T. D. Anthopoulos, "Recent Progress in High-Mobility Organic Transistors: A Reality Check," Adv. Mater., pp. 1801079, Jul 18, 2018, doi:10.1002/adma.201801079.

[2] C. Liu, G. Li, R. Di Pietro, J. Huang, Y.-Y. Noh, X. Liu, and T. Minari, "Device Physics of Contact Issues for the Overestimation and Underestimation of Carrier Mobility in Field-Effect Transistors," Phys. Rev. Appl., vol. 8, no. 3, 2017, doi:10.1103/PhysRevApplied.8.034020.

[3] W. Shockley, "A Unipolar "Field-Effect" Transistor," Proceedings of the IRE, vol. 40, no. 11, pp. 1365-1376, 1952, doi:10.1109/JRPROC.1952.273964.

[4] Y. Xu, H. Sun, A. Liu, H.-H. Zhu, W. Li, Y.-F. Lin, and Y.-Y. Noh, "Doping: A Key Enabler for Organic Transistors," Adv. Mater., vol. 0, no. 0, pp. 1801830, doi:doi:10.1002/adma.201801830.

[5] H. Klauk, "Organic thin-film transistors," Chem. Soc. Rev. , vol. 39, no. 7, pp. 2643-66, Jul, 2010, doi:10.1039/b909902f.

[6] C. Reese, and Z. Bao, "Overestimation of the field-effect mobility via transconductance measurements and the origin of the output/transfer characteristic discrepancy in organic field-effect transistors," J. Appl. Phys., vol. 105, no. 2, pp. 024506, 2009, doi:10.1063/1.3029587.

[7] Y. Hu, G. Li, and Z. Chen, "The Importance of Contact Resistance in High-Mobility Organic Field-Effect Transistors Studied by Scanning Kelvin Probe Microscopy," IEEE Electron Device Lett., vol. 39, no. 2, pp. 276-279, 2018, doi:10.1109/LED.2017.2781301. 
[8] G. Ghibaudo, "New method for the extraction of MOSFET parameters," Electronics Letters, vol. 24, no. 9, pp. 543, 1988, doi:10.1049/el:19880369.

[9] Y. Xu, H. Sun, A. Liu, H. Zhu, B. Li, T. Minari, F. Balestra, G. Ghibaudo, and Y.-Y. Noh, "Essential Effects on the Mobility Extraction Reliability for Organic Transistors," Adv. Funct. Mater., pp. 1803907, 2018, doi:10.1002/adfm.201803907.

[10] I. McCulloch, A. Salleo, and M. Chabinyc, "Avoid the kinks when measuring mobility," Science, vol. 352, no. 6293, pp. 1521-1522, 2016, doi:10.1126/science.aaf9062.

[11] Y. Xu, T. Minari, K. Tsukagoshi, J. A. Chroboczek, and G. Ghibaudo, "Direct evaluation of low-field mobility and access resistance in pentacene field-effect transistors," J. Appl. Phys., vol. 107, no. 11, pp. 114507, 2010, doi:10.1063/1.3432716.

[12] W. Zhang, J. Smith, S. E. Watkins, R. Gysel, M. McGehee, A. Salleo, J. Kirkpatrick, S. Ashraf, T. Anthopoulos, M. Heeney, and I. McCulloch, "Indacenodithiophene Semiconducting Polymers for High-Performance, Air-Stable Transistors," J. Am. Chem. Soc., vol. 132, no. 33, pp. 11437-11439, 2010/08/25, 2010, doi:10.1021/ja1049324.

[13] D. Venkateshvaran, M. Nikolka, A. Sadhanala, V. Lemaur, M. Zelazny, M. Kepa, M. Hurhangee, A. J. Kronemeijer, V. Pecunia, I. Nasrallah, I. Romanov, K. Broch, I. McCulloch, D. Emin, Y. Olivier, J. Cornil, D. Beljonne, and H. Sirringhaus, "Approaching disorder-free transport in high-mobility conjugated polymers," Nature, vol. 515, no. 7527, pp. 384-8, Nov 20, 2014, doi:10.1038/nature13854.

[14] J. Lee, J. W. Chung, D. H. Kim, B. L. Lee, J. I. Park, S. Lee, R. Hausermann, B. Batlogg, S. S. Lee, I. Choi, I. W. Kim, and M. S. Kang, "Thin Films of Highly Planar Semiconductor Polymers Exhibiting Band-like Transport at Room Temperature," J. Am. Chem. Soc., vol. 137, no. 25, pp. 7990-3, Jul 1, 2015, doi:10.1021/jacs.5b04253.

[15] S. Sze, "Physics of semiconductor devicesWiley Eastern," New York, 1981,

[16] Y. Xu, H. Sun, W. Li, Y. F. Lin, F. Balestra, G. Ghibaudo, and Y. Y. Noh, "Exploring the Charge Transport in Conjugated Polymers," Adv. Mater., vol. 29, no. 41, Nov, 2017, doi:10.1002/adma.201702729.

[17] T. Sakanoue, and H. Sirringhaus, "Band-like temperature dependence of mobility in a solution-processed organic semiconductor," Nat. Mater., vol. 9, pp. 736, 08/22/online, 2010, doi:10.1038/nmat 2825

[18] E. G. Bittle, J. I. Basham, T. N. Jackson, O. D. Jurchescu, and D. J. Gundlach, "Mobility overestimation due to gated contacts in organic field-effect transistors," Nat. Commun., vol. 7, pp. 10908, Mar 10, 2016, doi:10.1038/ncomms 10908.

[19] T. Uemura, C. Rolin, T. H. Ke, P. Fesenko, J. Genoe, P. Heremans, and J. Takeya, "On the Extraction of Charge Carrier Mobility in High-Mobility Organic Transistors," Adv. Mater., vol. 28, no. 1, pp. 151-5, Jan 6, 2016, doi:10.1002/adma.201503133.

[20] Y. Xu, H. Sun, E. Y. Shin, Y. F. Lin, W. Li, and Y. Y. Noh, "Planar-Processed Polymer Transistors," Adv. Mater., vol. 28, no. 38, pp. 8531-8537, Oct, 2016,

[21] Y. Hu, G. Li, W. Peng, and Z. Chen, "Comparing the Gate Dependence of Contact Resistance and Channel Resistance in Organic Field-Effect Transistors for Understanding the Mobility Overestimation Issue," IEEE Electron Device Lett., vol. 39, no. 3, pp. 421-423, 2018, doi:10.1109/LED.2018.2798288.

[22] C. Liu, Y. Xu, and Y.-Y. Noh, "Contact engineering in organic field-effect transistors," Materials Today, vol. 18, no. 2, pp. 79-96, 2015/03/01/,2015, doi:https://doi.org/10.1016/j.mattod.2014.08.037.

[23] J. Z. Wang, Z. H. Zheng, and H. Sirringhaus, "Suppression of short-channel effects in organic thin-film transistors," Appl. Phys. Lett., vol. 89, no. 8, pp. 083513, 2006, doi:10.1063/1.2234724. 